\title{
Twenty years of bacterial RNases and RNA processing: how we've matured
}

\author{
MURRAY P. DEUTSCHER \\ Department of Biochemistry and Molecular Biology, University of Miami Miller School of Medicine, Miami, Florida 33103, USA
}

By 1995, the year that publication of RNA began, the field of RNA processing was already well established. A dedicated cadre of scientists was actively engaged in studying how RNA precursors are converted to their mature, functional forms, and conferences dealing with the topic were held at regular intervals. In fact, members of the RNA processing community were instrumental in founding the RNA Society and starting $R N A$. What follows are the personal reflections and biases of one who has worked in the RNA processing field from very early on. Emphasis will be on advances in bacterial systems, with which I am most familiar, but readers should be aware that the last 20 years has seen an explosion of information about RNA metabolism in eukaryotic systems, leading to the realization that organisms from bacteria to mammals share much in common in their RNA metabolic processes. As a consequence, information gleaned in one organism has had important ramifications for studies in all systems.

\section{The way we were (in 1995)}

The general outlines of bacterial RNA maturation were already apparent in 1995 . Thus, rRNAs were known to be transcribed as a single $30 \mathrm{~S}$ RNA that was subsequently converted to the individual $16 \mathrm{~S}, 23 \mathrm{~S}$ and $5 \mathrm{~S}$ RNAs by a series of largely uncharacterized processing reactions. tRNAs were transcribed as part of multimeric or individual precursor molecules or, sometimes, together with rRNAs or mRNAs. Processing reactions converted these molecules first to individual precursors followed by removal of the extra $5^{\prime}$ and $3^{\prime}$ residues. In Escherichia coli, no additional maturation was required as the universal 3'-CCA sequence was already encoded. What generally was not known was the order of the processing reactions, whether the reaction order was obligatory, and often, the ribonucleases (RNases) involved. It was assumed that the processing pathways and RNases involved, as identified in E. coli, were applicable to other bacteria as well.

\footnotetext{
Corresponding author: mdeutsch@med.miami.edu

Article and publication date are at http://www.rnajournal.org/cgi/doi/ 10.1261/rna.049692.115. Freely available online through the RNA Open Access option.
}

With regard to the RNases, work in E. coli over many years had identified and characterized about 15 distinct proteins with ribonuclease activity. Relatively little was known about RNases in other bacteria, and what information was available, primarily from Bacillus subtilis, suggested that its RNase content was highly similar to that of $E$. coli, although a few differences (such as the absence of RNase II in B. subtilis) had begun to emerge. Despite the identification of so many RNases in a single cell, the function of many of them was unclear. It was known that the endoribonuclease, RNase III, cleaved the initial rRNA transcript to generate precursors of the individual rRNAs, and that another endoribonuclease, RNase P, was responsible for generating the $5^{\prime}$ end of tRNA. Multiple exoribonucleases (RNases T, PH, D, II and BN) could process the $3^{\prime}$ end of tRNA, but why so many were involved was unknown. RNase $\mathrm{T}$ was about to be shown to be required for $3^{\prime}$ processing of 5S RNA. Two exoribonucleases, RNase II and polynucleotide phosphorylase (PNPase) had been identified as participating in mRNA degradation, and the absence of both enzymes was found to be lethal. Finally, the endoribonuclease, RNases E, had just been shown to be an important participant in the initial breakdown of mRNAs. This was an unexpected finding because RNase E was already known to be involved in 5S rRNA maturation, and the prevailing view at the time was that distinct RNases would be used for unstable mRNA decay and for stable RNA processing. In fact, it is now understood that there is widespread overlap in the RNases used for all RNA metabolic processes, and that only the specificity of the RNases and the accessibility of the substrate determine whether an RNA molecule will be acted upon.

In the ensuing paragraphs, I will describe what I believe have been some of the more important advances that have been made in the field of RNases and RNA processing since the founding of $R N A$, much of which has appeared in the pages of this journal. These advances have strongly influenced my thinking, and have profoundly affected how the field has developed over the past 20 years.

(C) 2015 Deutscher This article, published in RNA, is available under a Creative Commons License (Attribution-NonCommercial 4.0 International), as described at http://creativecommons.org/licenses/by-nc/4.0/. 


\section{There's more to life than $E$. coli}

As our knowledge about RNases and RNA metabolism expanded, it became clear that not all bacterial systems are like E. coli. In particular, studies of the Gram-positive organism, B. subtilis, revealed a number of important differences, both in the RNases present and in RNA metabolic pathways. First, sequencing of the B. subtilis genome indicated that, in addition to the absence of RNase II, the essential E. coli enzymes, RNase E and oligoribonuclease, are also not found, immediately suggesting the likelihood of differences in mRNA turnover and rRNA processing. Secondly, since approximately one-third of $B$. subtilis tRNA precursors lack an encoded -CCA sequence, it was likely that $3^{\prime}$ tRNA processing also differed from that of E. coli. Probably, the most exciting development was the discovery of RNase J1, the first bacterial $5^{\prime}$ to $3^{\prime}$ exoribonuclease. While enzymes with $5^{\prime}$ to $3^{\prime}$ specificity were well known in eukaryotic cells, they had not been identified in bacteria despite considerable effort and the existence of reactions that seemed to call for such an activity.

All of this information, together with many additional studies, led to the current realization that RNA metabolism in B. subtilis, while similar overall, differs in significant details from that of $E$. coli. Thus, we now know that $5^{\prime}$ processing of $16 \mathrm{~S}$ rRNA in B. subtilis uses the $5^{\prime}$ exoribonuclease, $\mathrm{J} 1$, in contrast to $E$. coli which carries out a two-step process involving RNases E and G. Additionally, for $3^{\prime}$ maturation of those tRNA precursors lacking an encoded -CCA sequence, the process requires an endonucleolytic cleavage by RNase $\mathrm{Z}$ followed by addition of the -CCA by tRNA nucleotidyltransferase. Interestingly, for those tRNA precursors that encode a -CCA, the extra $3^{\prime}$ residues are removed by RNase $\mathrm{PH}$, as in E. coli. The process differs slightly because B. subtilis lacks RNase $\mathrm{T}$ which plays a significant role in E. coli. As noted, turnover of some mRNAs also differs between the two organisms. While one pathway is similar to E. coli, using endonucleolytic cleavage by RNase Y instead of RNase E, B. subtilis also employs RNase J1 for direct degradation of messages from the $5^{\prime}$ end.

From examples such as these, we now appreciate that diverse organisms do not all follow the pathways first observed in E. coli nor is their repertoire of RNases the same. In fact, when one considers Mycoplasma, they manage to carry out all their processing and degradative reactions with an RNase complement that consists of only RNases III, P, M5, H3, R and nanoRNase. Undoubtedly, as studies of RNA metabolism expand into more and more organisms, we can look forward to additional surprises.

\section{What's behind reaction order in RNA processing?}

A major focus of studies of RNA processing early on was to define the order of processing reactions en route to the mature RNA. However, as our insights into RNA processing advanced, our thinking has become much more nuanced. We now realize that RNases act on any RNA to which they have access and which fits within the RNases' specificity. Consequently, multiple RNases may be able to carry out the same processing reaction, and which one does will depend simply on which one binds and acts first. This occurs in the $3^{\prime}$ processing of E. coli tRNAs and $16 \mathrm{~S}$ rRNA in which the presence of any one of multiple exoribonucleases is sufficient to allow $3^{\prime}$ maturation to proceed.

The same reasoning applies to the order of processing reactions. The order is determined by which one of multiple RNases binds and acts first, whether it is at the $5^{\prime}$ terminus, the $3^{\prime}$ terminus, or internally on an RNA molecule. In fact, two identical RNA precursors might undergo a different sequence of processing reactions depending on which RNase acts first on each precursor. For other precursors, a preferred order may predominate because the RNA structure precludes action on one end of the RNA. As an example, $3^{\prime}$ processing of $16 \mathrm{~S}$ rRNA precedes maturation of the $5^{\prime}$ terminus because RNase E cannot cleave at residue +66 in the $5^{\prime}$ precursor region due to its location within a double-stranded stem. Only when $3^{\prime}$ maturation removes the complementary strand is the single strand-specific RNase E able to act. Thus, the order of RNA processing is built into the structure of the precursor and the specificity of the RNases. This understanding represents a significant advance in our thinking over the last 20 years.

\section{Quality control eliminates mistakes}

It has long been appreciated that processes that synthesize, mature and assemble RNA molecules into RNP particles are not perfect, and that mistakes occur. For mRNA, its rapid turnover effectively eliminates defective molecules, but even in this case, trans-translation and other processes are needed to degrade defective messages on ribosomes and remove their translation products. But, what about the stable RNAs, rRNA and tRNA? These molecules also can accumulate errors in sequence, folding, and/or assembly, with serious consequences for protein synthesis.

Several studies in recent years identified quality control processes that have greatly increased our understanding of how cells deal with defective RNA molecules. These processes eliminate defective tRNAs, partially assembled ribosomes and even completed ribosomes. Thus, a mutant tRNA that folds improperly is efficiently degraded leading to a more than $80 \%$ reduction in its steady-state level. Most interestingly, such degradation occurs at the precursor level and is dependent on prior polyadenylation of the defective precursor. RNase R and PNPase are primarily responsible for the degradation, and the polyadenylation is required to provide a binding site for the RNase. The same two RNases are responsible for degrading rRNA when ribosomes are assembling incorrectly. In the absence of these two RNases, massive amounts of large rRNA fragments accumulate, ultimately leading to cell death. The endoribonuclease responsible for generating the rRNA fragments is not known. While this 
quality control process acts on assembling ribosomes, another process acts on fully-assembled, defective $70 \mathrm{~S}$ ribosomes using the protein, YbeY, and the exoribonuclease, RNase R.

Although the existence of quality control processes to eliminate defective RNAs is now accepted, the mechanisms by which RNases recognize defective substrates are largely unknown. While tRNAs and ribosomes are generally stable due to aminoacylation and a compact, tertiary structure for tRNAs, or as part of an RNP particle for rRNA, their defective counterparts are readily degraded. Most likely, these defective molecules have undergone some change that allows an RNase to initiate degradation. One can imagine, for example, that a defective tRNA might be poorly aminoacylated leaving its $3^{\prime} \mathrm{OH}$ terminus exposed. Similarly, an incorrectly assembling or assembled ribosome might have exposed RNA sequences that could be cleaved by an endoribonuclease. Despite the lack of details, the concept of quality control of RNA is now firmly ingrained in our thinking, and new information is sure to follow.

\section{RNases can be regulated}

RNases play an essential role in almost every aspect of RNA metabolism, but they can also be destructive enzymes that need to be controlled. Moreover, RNases may have to respond to changes in cell physiology that require up- or down-regulation. The idea that RNases might be regulated was just beginning to gain some traction when $R N A$ was founded based on studies of autoregulation of PNPase, RNaseIII and RNase $\mathrm{E}$, but there was relatively little follow-up on how this regulation relates to RNA metabolism and to cell physiology. My perspective on the importance of RNase regulation has been strongly influenced by recent studies from our lab on regulation of the exoribonuclease, RNase $\mathrm{R}$.

Levels of RNase R increase three- to 10-fold under conditions of stress such as stationary phase or cold shock. RNase R is also an extremely unstable protein with a half-life of $10 \mathrm{~min}$ in exponential phase cells. The elevation of RNase R during stress is entirely due to its stabilization such that it no longer is degraded under these conditions. The instability of RNase $\mathrm{R}$ is dependent on binding of two trans-translation factors, tmRNA and SmpB, to the C-terminal region of the exponential phase protein. Binding to the stationary phase enzyme is much weaker, and this difference is due to the presence of a single acetylated lysine in the exponential phase, but not the stationary phase, enzyme. Only the exponential phase RNase $\mathrm{R}$ is acetylated because the acetylating enzyme, termed Pka, is not present in stationary phase cells. Exponential phase RNase $\mathrm{R}$ is subject to degradation because $\mathrm{SmpB}$ bound at its C-terminal region enhances binding of the proteases, Lon or HslUV, to its N-terminal region leading to proteolysis. Thus, a series of events initiated by acetylation leads to instability of exponential phase RNase R. Since stationary phase RNase $\mathrm{R}$ is not acetylated, it is stable. An identical situation occurs upon cold shock.
RNase R stability is also affected by ribosomes. Approximately $80 \%$ of RNase R is bound to ribosomes in exponential phase cells where it is stable and participates in trans-translation. Free RNase R is extremely unstable, turning over with a half-life of $2 \mathrm{~min}$. The free form is also deleterious to cells; elevation of the free form by inhibiting its binding to ribosomes leads to slower growth and a large increase in stable RNA degradation. Thus, sequestration of RNase R serves to protect RNA in growing cells. In contrast, in stationary phase cells all of the RNase R is free, and as noted above, is also stable. In this phase of growth, an increase in stable RNA degradation is likely to be beneficial as protein synthesis is much less active, and ribosomes represent a large storehouse of nutrients that could be used.

Although the complexity of RNase R regulation was quite surprising, from a mechanistic perspective, it provided the first examples of acetylation and binding to ribosomes affecting the stability of a bacterial protein. Physiologically, it is now clear that the levels of an RNase may be adjusted to the metabolic demands of a cell. These findings have dramatically altered my previous ideas of RNases as constitutive enzymes indiscriminately acting on susceptible RNAs. Moreover, regulation of RNase R is unlikely to be unique, but likely will extend to other RNases as well. In fact, recent work from our laboratory has confirmed this point ( $\mathrm{T}$ Dutta and MP Deutscher, unpubl.).

\section{Other important advances}

Space limitations prevent detailed discussion of other important advances in the RNase field over the past 20 years, but I must mention several: a) RNases can be organized into multienzyme complexes such as the degradosome and the eukaryotic exosome; b) RNases may be localized as has been found for the membrane association of the degradosome, RNase Y, and RNase II, and for other RNases that are present in structures that coil around the cell periphery; c) Structural analysis of many RNases has revealed common features of multiple RNase families and has provided a structural framework for analysis of RNase catalytic specificity and mechanism.

\section{Where do we go from here?}

Although additional, distinct RNases may yet be discovered, particularly as we study more diverse bacteria, I think that the repertoire of RNases in E. coli and B. subtilis is nearing completion. Most major reactions of RNA metabolism have already been associated with one or more RNases, and for those reactions still in need of an RNase, the activities of known RNases may suffice, as was recently found for the $3^{\prime}$ end of $16 \mathrm{~S}$ rRNA. On the other hand, much remains to be learned about RNase regulation and how RNase activities are coordinated with the physiological state of the cell. I am also intrigued by the recent work on RNase association 
with the cell membrane. Are these storage sites to be released as needs arise or are they functional sites? If the latter, does this mean that RNA precursors shuttle to the membrane and that RNAs to be degraded must be transferred to the cell membrane? How does RNase association with the membrane change with cell physiology? Similar questions arise for the degradosome. It is already known that its composition can change under certain conditions. How extensive is this and how many degradosome states are possible? All of these questions are amenable to study and ready to be tackled.

Studies will also continue on RNase structure, particularly as it relates to enzyme mechanism. RNases display a wide range of substrate specificities. How is this is accomplished?
Also related to RNase structure is post-translational modification. Bacterial RNases are already known to be phosphorylated and acetylated. What other modifications may exist and how might they change in relation to cell physiology? These are only a few possibilities as we continue the study of RNases, but there is no doubt that the next 20 years will prove to be as exciting and interesting as the past 20 .

\section{Acknowledgments}

Work from the author's laboratory was supported by Grant GM16317 from the National Institutes of Health. 

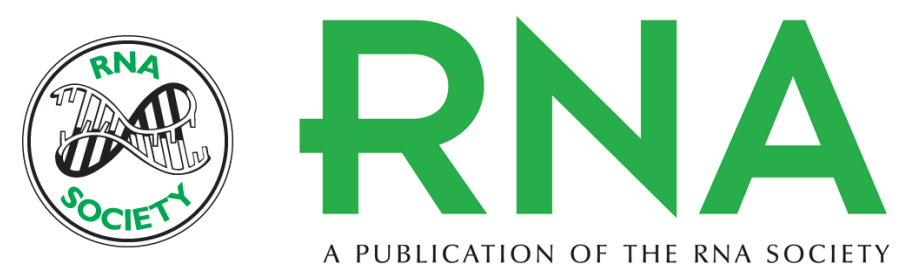

A PUBLICATION OF THE RNA SOCIETY

\section{Twenty years of bacterial RNases and RNA processing: how we've matured}

Murray P. Deutscher

RNA 2015 21: 597-600

Open Access Freely available online through the RNA Open Access option.

Creative This article, published in RNA, is available under a Creative Commons License

Commons (Attribution-NonCommercial 4.0 International), as described at

License http://creativecommons.org/licenses/by-nc/4.0/.

Email Alerting Receive free email alerts when new articles cite this article - sign up in the box at the Service top right corner of the article or click here. 